GANIT J. Bangladesh Math. Soc. (ISSN 1606-3694) Vol. 33 (2013) 41 - 51

\title{
ANALYTICAL STUDY OF INFLUENCE OF CHEMICAL AGENTS IN AGRICULTURAL SYSTEMS
}

\author{
M. Islam ${ }^{1}$, M. S. Uddin ${ }^{2}$, B.Islam ${ }^{3}$, N. Islam ${ }^{4}$ and H.P. Mazumdar ${ }^{5}$ \\ ${ }^{1}$ Department of Mathematics, Jadavpur University, Kolkata-700032, India \\ ${ }^{2}$ Mathematics Discipline, Khulna University, Khulna-9208, Bangladesh \\ ${ }^{3}$ Indian Statistical Institute, 203 B.T.Road, Kolkata -700108, India \\ ${ }^{4}$ Dept of Mathematics, Ramakrishna Miss. Res. College Kolkata-700103 \\ ${ }^{5}$ Physics and Applied Math Unit, Indian Statistical Institute, Kolkata -700108 \\ E-mail: ${ }^{1}$ mitul.islam@gmail.com, ${ }^{2}$ sharif_mathku@yahoo.com, ${ }^{3}$ mib.speaking@gmail.com
}

Received 15-09-2012 Accepted 30-10-2013

\begin{abstract}
Modern day agriculture is dependent on the use of chemical agents for maximizing crop yield. This practice has perilous effects on the ecosystem if used in an unchecked manner. The present paper develops a mathematical model of an agricultural system that incorporates the cumulative effect of the chemical agents like pesticides, fertilizers etc. Dynamical behaviour of the system, such as boundedness, permanence and stability, are studied. Numerical simulations are carried out to study the changes in the behaviour of the system due to varying levels of potency of the chemicals. Based on these, an effort is made to determine the conditions necessary for a sustainable and productive agricultural system in presence of chemical agents. Applicability of the model in related scenarios is also discussed.
\end{abstract}

Keywords: Prey-predator model, Permanence; Sustainability; productivity, Poincaré-Bendixson theorem, Global stability.

\section{Introduction}

According to a quote attributed to Charles Darwin, the limiting factor of growth of each species is not only the food available to it but also the inter-dependence among various existing species which can be classified as predators and preys. This maintains ecological balance and determines the average numbers of a species. In higher food-chain this preypredator relationship is pronounced as the interdependence becomes a key factor for sustenance of the dominating species. Often multiple predator species may be vying for a common food source. One perfect example fitting the bill is the competition between humans and pests for food in form of agricultural yield. A major food source for humans is agricultural production and plant pests are a single most significant factor that affects both yield and quality of this food source.

Humans have tried to combat this scene using chemical agents. However humans have tried a two-pronged attack, one, by introducing pesticides, and two, by using fertilizers. Pesticides solely target in killing off or check the growth of pests in the agricultural scenario, while fertilizers aim to improve the net yield and also the quality of agricultural production. 
However unrestricted use of both classes of chemicals can be damaging to the ecosystem. Problems like development of resistance in the pest population, poisonous effect on human health, denaturing of natural soil quality, spread of chemicals into the ecosystem through other predators interacting with pests and plants [4]. Thus, though the system of chemical pest control is extremely widespread, there is much concern about the negative effects of use of chemicals. However, it cannot be denied that use of chemicals is by far the most prevalent and cost effective method of pest control. In this situation, we try to determine whether it is possible to have an agricultural system that is 'profitable' and 'sustainable', even after we add chemical agents.

In theoretical ecology, several theoretical pest control models [15] exist and they have been extensively studied mathematically, e.g. integrated pest management (IPM) [17], sterile insect release method (SIRM) [6,14], microbial pest control [2,13], impulsive control strategies [18], etc. On the other hand, the effect of infected pests or infected predators in the classical prey-predator model $[1,5,9]$ have been thoroughly studied under eco-epidemiology. This paper analytically studies the method of pest control using chemicals. We study the impact of the two class of chemicals- fertilizers and pesticideson a non-linear system consisting of crops, pests, pesticides with varying degree of effectiveness against the pest and fertilizer compounds with varying effectiveness for crop yield. A modified classical prey-predator model simulates the interaction of the four aspects of the system and we investigate the various conditions that would be necessary for a sustainable and productive agricultural scenario. Subsequently we elaborate our model and perform various analyses on the system.

\section{The Mathematical Model}

Agricultural systems can be effectively modeled by using modified versions of the classical prey-predator model. Agricultural systems commonly comprise of a crop population, grown to satisfy human needs and hence harvested from time to time, and a pest population, that feeds on the crops and hence degrades the quality and quantity of the agricultural produce. Hence, human beings add chemical agents to the system so as to protect the preys ,or the crops, from the predators, or the pests. In this model, we broadly classify all types of chemicals into two groups - fertilizers and pesticides. We now introduce two parameters $\gamma$ and $\theta$ both of which lie in the interval $[0,1)$. These parameters measure the effectiveness or potency of the respective chemicals. Greater the value of $\gamma$, more is the ability of the fertilizer to promote plant growth and survival. Greater the value of $\theta$, more is the toxicity and lethality of the pesticide towards the predators.

The crop population, given by $x(t)$, is assumed to grow logistically, with intrinsic growth rate $r(>0)$ and environmental carrying capacity $K_{1}$, in absence of any chemical agents or the predators. The predation is assumed to follow the law of mass action, with $\beta$ being the predation parameter. The harvesting of crops is realized in the model by adding the prey harvesting term, with $h(>0)$ being the constant harvesting rate. To this classical equation for growth of preys, we now add the effects of chemical agents. 
Addition of fertilizers increases the presence of vital minerals and chemicals in the soil and help in plant growth. Thus we assume that presence of fertilizers enhance the intrinsic growth rate of the preys, the resulting increase being directly proportional to the potency of the fertilizer. The intrinsic growth rate is modified to $r(\gamma)=r(1+\gamma)$. The availability of vital resources due to addition of fertilizers increases the environmental carrying capacity according to the relation $k^{\prime}=k_{1} /(1-\gamma)$. The pesticides, on the other hand, is assumed to weaken the predators and/or make them more cautious during predation. Thus the predation parameter gets modified into $\beta(\theta, n)=\beta\{1-(\theta / n)\} ;(n>1)$. For the current model, we assume $n=2$ and this choice is purely arbitrary. It is an established fact that though pesticides are necessary for protecting the crops, still these chemicals 'poison' the environment to some degree [1] .This is implemented in the model by lowering the carrying capacity of preys; the decrease depending on the parameter $\theta$. The modified carrying capacity is $K=k^{\prime}(1-\theta)=k_{1}(1-\theta) /(1-\gamma)$. Thus, the equation governing growth of preys comes out to be

$$
\frac{d X}{d t}=r(1+\gamma) X(1-X / K)-\beta(1-\theta / 2) X Y-h X
$$

The pest population, given by $y(t)$, is assumed to be entirely dependent on the crops for their growth and survival. This dependence is assumed to follow the law of mass action, with $a \in(0,1)$ as the conversion rate, and is given by the term $a \beta\{1-(\theta / 2)\} X Y$. In absence of any chemical agents, the pests have a natural death rate $d_{1}$. However, the presence of pesticide in the environment means a portion of it enters the body of the predators through the body of the preys. These chemicals are suitably designed such that they can selectively attack only the predators. Predation thus infects ( or poisons) the predators, with $\theta$ determining the severity of this 'poisoning', and gives rise to the term $\beta \theta X Y$. The growth of predators is thus modeled as :

$$
\frac{d Y}{d t}=a \beta(1-\theta / 2) X Y-\beta \theta X Y-d_{1} Y
$$

An agricultural system, in presence of chemical agents, can thus be modeled by the following system of ordinary differential equations:

$$
\begin{aligned}
& \frac{d X}{d t}=r(1+\gamma) X(1-X / K)-\beta(1-\theta / 2) X Y-h X \\
& \frac{d Y}{d t}=a \beta(1-\theta / 2) X Y-\beta \theta X Y-d_{1} Y
\end{aligned}
$$

with $K=k_{1}(1-\theta) /(1-\gamma)$ and the initial conditions $X(0)>0$ and $Y(0) \geq 0$. 


\section{Equilibrium points and their existence}

The system possess a trivial equilibrium point $(0,0)$, which always exists. The axial equilibrium point $E_{A}\left(X_{2}, Y_{2}\right)$ is given by $X_{2}=K\{1-(h /[r(1+\gamma)])\}$ and $Y_{2}=0$. It exists, provided $\gamma>(h / r)-1$.

The interior equilibrium point of the system is $E^{*}\left(X^{*}, Y^{*}\right)$; where $X^{*}=d_{1} / \beta\{a(1-\theta / 2)-\theta\}$ and $Y^{*}=\left[r(1+\gamma)\left\{1-\left(X^{*} / K\right)\right\}-h\right] / \beta(1-\theta / 2)$

Conditions for existence of $X^{*}$ and $Y *$ are:

$$
\begin{aligned}
& 2 a /(a+2)>\theta \\
& 1-h / r(1+\gamma)>d_{1} / K \beta\{a(1-\theta / 2)-\theta\}>0
\end{aligned}
$$

\section{Boundedness}

Boundedness of a system implies that all trajectories of the system are present within a compact set. A system that is bounded is always biologically well behaved.

Theorem 4.1 All solutions of the system starting in $\mathfrak{R}_{+}^{2}$ are bounded.

Proof. Let $(X(t), Y(t))$ be any solution of the system in $\mathfrak{R}_{+}^{2}$. We consider a function

$$
W(t)=X(t)+\{Y(t) / a\}
$$

Thus,

$$
\frac{d W(t)}{d t}=\frac{d X(t)}{d t}+(1 / a) \frac{d Y(t)}{d t} \leq r(1+\gamma) X-h X-\left(d_{1} / a\right) Y \leq M_{1}-M_{2} W
$$

Where, $M_{1}=r(1+\gamma) \delta, \delta=\max \{X(0), k\}, M_{2}=\min \left\{h, d_{1}\right\}$

Applying a theorem on differential inequalities [12], we obtain,

$$
\begin{gathered}
0 \leq W(W, Y) \leq\left(M_{1} / M_{2}\right)+W(X(0), Y(0) / a) \exp \left(-M_{2} t\right) \text { and } \\
\text { for } t \rightarrow \infty, 0 \leq W \leq\left(M_{1} / M_{2}\right) .
\end{gathered}
$$

Thus, all solutions of the system enter into the compact set:

$$
\Omega=\left\{(X, Y): 0 \leq W \leq\left(M_{1} / M_{2}\right)+\in \text { for any } \in>0\right\}
$$

\section{Permanence}

A system is permanent when its boundary equilibrium are repellers. The permanence of a system can be proved using "Average Liapunov" functions [13].

Theorem 5.1 The system is permanent provided

$$
1-h / r(1+\gamma)>d_{1} /[K \beta\{a(1-\theta / 2)-\theta\}]
$$


Proof. Let us consider an average Lyapunov function of the form $V(X, Y)=X^{\alpha_{1}} Y^{\alpha_{2}}$, where $\alpha_{1}$ and $\alpha_{2}$ are positive. In the interior of $\mathfrak{R}_{+}^{2}$, we have,

$$
\begin{aligned}
& \dot{V} / V=f(X, Y) \\
& =\alpha_{1}\{r(1+\gamma)(1-X / K)-\beta(1-\theta / 2) Y-h\}+\alpha_{2}\left\{a \beta(1-\theta / 2) X-\beta \theta X-d_{1}\right\}
\end{aligned}
$$

For the system to be permanent, $f(X, Y)>0$ for all boundary equilibiria of the system. Thus the axial equilibrium point $E_{A}\left(X_{2}, Y_{2}\right)$ should satisfy:

$f\left(X_{2}, Y_{2}\right)=\alpha_{2} \beta\{a(1-\theta / 2)-\theta\} X_{2}-\alpha_{2} d_{1}>0$

Hence, the theorem.

\section{Local stability Analysis}

The variational matrix $V(E)$ at an equilibrium point $E(X, Y)$ of the system is given by,

$$
V(E)=\left(\begin{array}{ll}
p_{11} & p_{12} \\
p_{21} & p_{22}
\end{array}\right)
$$

Where,

$$
\begin{aligned}
& p_{11}=r(1+\gamma)(1-2 X / K)-\beta(1-\theta / 2) Y-h, p_{12}=-\beta(1-\theta / 2) X \\
& p_{21}=\alpha \beta(1-\theta / 2) Y-\beta \theta Y, p_{22}=\alpha \beta(1-\theta / 2) X-\beta \theta X-d_{1}
\end{aligned}
$$

The characteristic equation is,

$$
\lambda^{2}+a \lambda+b=0
$$

where $a=-\left(p_{11}+p_{22}\right)$ and $b=p_{11} p_{22}-p_{12} p_{21}$.

Lemma 6.1 The system is locally asymptotically stable around $E(X, Y)$ when $a>0$ and $b>0$

Lemma 6.2 The system is stable, but not asymptotically [14] when

1. $\mathrm{a}>0$ and $\mathrm{b}=0$, or,

2. $a=0$ and $b>0$,

Otherwise the system is unstable around $E(X, Y)$.

Determining the corresponding characteristic equations for the three equilibrium points and using lemmas 6.1 and 6.2 we may obtain the following result:

Theorem 6.3 The system is locally asymptotically stable around $(0,0)$ provided $(h / r)-1>\gamma$. 
Theorem 6.4 The system cannot be locally asymptotically stable around $E_{A}\left(X_{A}, Y_{A}\right)$, that is, $E_{A}$ can never be a stable node. $E_{A}$ is stable but not asymptotically when

$$
\{1-h / r(1+\gamma)\}\{r(1+\gamma)+\beta k(\theta+a \theta / 2-a)\}+d_{1}>0
$$

Theorem 6.5 $E^{*}\left(X^{*}, Y^{*}\right)$ is always a stable node. Existence of $\mathrm{E}^{*}$ ensure local asymptotic stability around $E^{*}$.

\section{Global Stability Analysis}

For studying global stability of a dynamical system, it is essential to know about the closed orbits of the system. An important theorem related to the context is the BendixsonDuLac theorem (or negative criterion) $[15,16]$, which states that if there exists a function $\mathrm{B}(\mathrm{X}, \mathrm{Y})$ such that

$$
\xi=\frac{\partial}{\partial x}(B X)+\frac{\partial}{\partial y}(B Y) \neq 0
$$

has the same sign almost everywhere in a simply connected region, then the plane autonomous system (3) has no periodic solutions or orbits. The term "almost everywhere" means 'everywhere', except in the set of measure ' 0 '. [15].

Lemma 7.1 A limit cycle cannot occur in the system (3).

Proof. Setting $B(X, Y)=1 /(X Y)$ gives.

$$
\xi=\frac{\partial}{\partial x}\left(\frac{1}{X Y} \dot{X}\right)+\frac{\partial}{\partial y}\left(\frac{1}{X Y} \dot{Y}\right)=\frac{r(1+\gamma)}{k} \frac{1}{Y}<0
$$

for all $Y \in \mathfrak{R}-\{0\}$.

Thus, $\xi<0$ for all $(X, Y) \in \Omega \subset \Re^{2}-\operatorname{span}\{(1,0)\}$ where $\operatorname{span}\{(a, b)\}$ is the subspace of $\mathfrak{R}^{2}$ spanned by (a,b). Applying Bendixson-Dulac theorem, it can now easily be proved that there are no periodic orbits.

Another important theorem concerning nature and behaviour of closed orbits is the Poincaré-Bendixson theorem [16,17]. It asserts that any orbit which stays in a bounded region of the phase plane at an autonomous system, either approaches a fixed point or a periodic orbit [15].

Lemma 7.2 Chaotic behaviour cannot arise in this model.

Proof. It is obvious from Poincaré-Bendixson theorem.

Theorem 7.3 Any solution of the system converges to the steady state equilibrium $\left(\mathrm{X}^{*}, \mathrm{Y}^{*}\right)$ as $t \rightarrow \infty$ provided $E^{*}$ exists.

Proof. Section 4 on Boundedness of the system proves that all solutions of the system are bounded. Thus using Poincaré-Bendixson theorem and lemma 7.1, it is obvious that closed orbits of the system must approach the interior equilibrium point whenever it exists. 


\section{Note:}

1. Existence of $E^{*}$ is a sufficient condition for existence of $E_{A}$.

2. Existence of $E^{*}$ is necessary and sufficient for permanence of the system.

3. For sufficiently low values of $\gamma$ and $\theta$ (so that we can neglect their higher powers), existence of $E^{*}$ reduces to

$$
1-d_{1} / a K \beta-h / r>\left(\theta d_{1} / a K \beta\right)(1 / 2+1 / a)-\gamma h / r
$$

\section{Sustainability and Productivity}

A sustainable ecosystem is "one whose practices can be maintained indefinitely without reducing the targeted species' ability to maintain its population at healthy levels, and without adversely impacting on other species within the ecosystem - including humans ", as defined by Greenpeace [18] . For our model (3) of the agricultural system, this criteria is mathematically equivalent to presence of the system in the vicinity of the interior equilibrium point $E^{*}$. From the viewpoint of human beings, sustainability of the system requires $E^{*}$ to be greater than $Y^{*}$.

In this context, productivity will be evaluated in terms of net crop output. If the net output obtained from the system after adding the chemicals is greater than that obtained without adding any chemicals, the system under action of chemicals will be termed profitable and more productive.

Thus, the necessary and sufficient conditions for an agricultural system to be sustainable and productive are:

1. Existence of $E^{*}$

2. Global asymptotic stability of the system around $E^{*}$

3. The system should be permanent

4. $X^{*}>Y^{*}$

5. Let both $X^{*}$ and $X^{*}$ be treated as functions of $\theta$ and $\gamma$. Then,

$$
X *(\theta, \gamma)>X *(0,0) \text { and } Y *(0,0)>Y *(\theta, \gamma)
$$

Theorem 8.1 For an agricultural system modelled by (3) to be sustainable and productive, the necessary and sufficient conditions are:

1. $2 a /(a+2)>\theta$

2. $1-h / r(1+\gamma)>d_{1} /[K \beta\{a(1-\theta / 2)-\theta\}]>0$

3.

$\left\{d_{1} / r(1+\gamma)\right\}\{\beta(1-\theta / 2)+r(1+\gamma) / K\}>\beta\{1-h / r(1+\gamma)\}$

$\{a(1-\theta / 2)-\theta\}>d_{1} / K$

4. $d_{1} /[K \beta\{a(1-\theta / 2)-\theta\}]-\left(d_{1} / \alpha \beta K_{1}\right)(1-\theta / 2)>\theta / 2(1-h / r(1+\gamma))$ 
Proof. Using the results obtained in Section 3, Section 5 and Section 7, conditions (i) and (ii) are obvious. Condition (iii) is obtained after some simplification of the inequality $X^{*}>Y^{*} . X^{*}(\theta, \gamma)>X^{*}(0,0)$ is always true. From the inequalities $Y *(0,0)>Y^{*}(\theta, \gamma)$, condition (iv) is obtained.
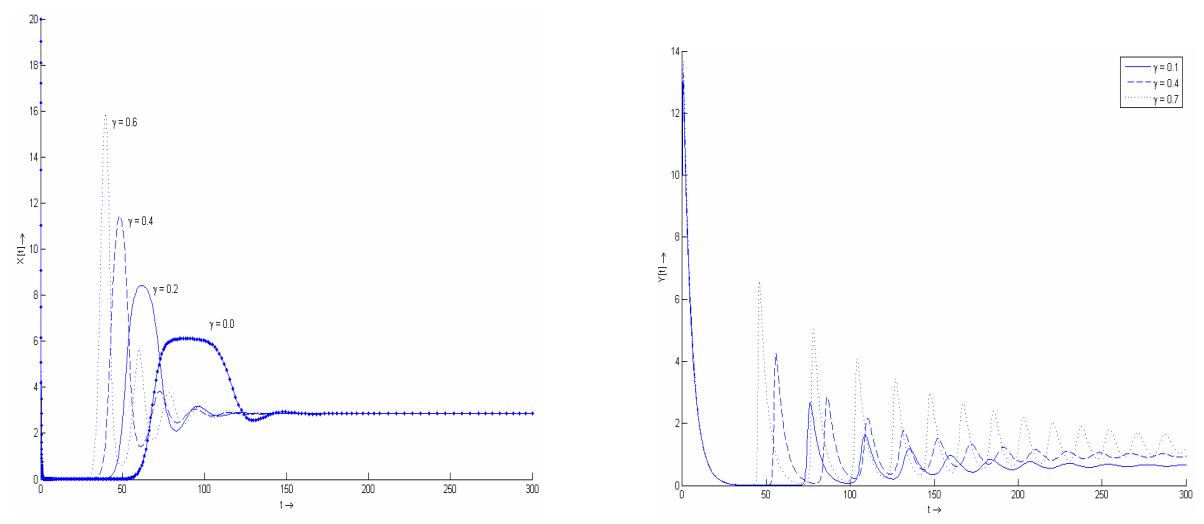

Fig. 1: $t \rightarrow X(t), \theta=0.5$, different $\gamma$ Fig. 2: $t \rightarrow X(t), \gamma=0.6$,different $\theta$
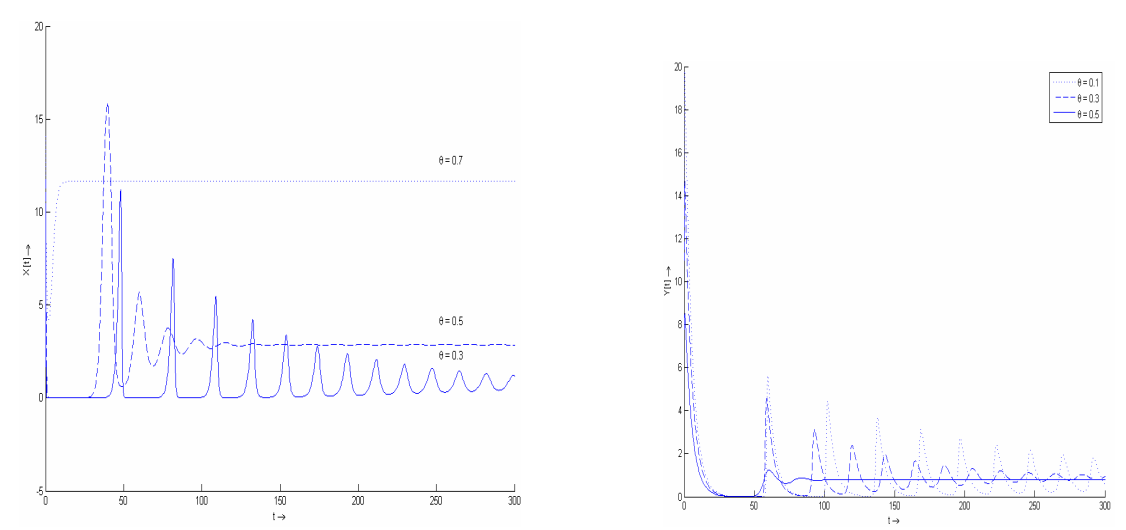

Fig $3 t \rightarrow Y(t), \theta=0.35$, different $\gamma$ Fig $4 t \rightarrow Y(t) \gamma=0.35$ different $\theta$ 


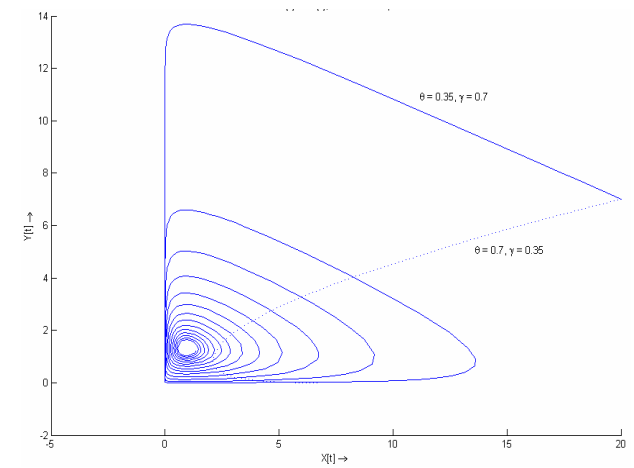

Fig 5 Phase portrait $\theta=0.35, \gamma=0.7$ and $\theta=0.7, \gamma=0.35$

\section{Conclusion}

Numerical simulation is carried out on system (3) with the initial conditions:

$X(0)=20, Y(0)=7 \quad K_{1}=21, r=0.6, \beta=0.7, h=0.25, a=0.8$ and $d_{1}=0.2$.

Numerical simulation of the model (3) brings out certain facts clearly. In absence of chemicals, that is, $\theta=0$ and $\gamma=0$, the model is the classical prey-predator model. For high values of $\theta$, irrespective of $\gamma$, the system reaches the axial equilibrium point $E_{A}$. Thus, we witness an extinction of the pests. From an ecological viewpoint, this is not at all appreciable though it might be a 'profitable' system. If $\gamma$ is varied while keeping $\theta$ fixed at some arbitrary moderate value (Fig. 1 and Fig. 2), the system reaches the interior equilibrium point $E^{*}$. The value of $\mathrm{X}^{*}$ increases with increase of $\gamma$, suggesting that use of fertilizers indeed enhance production. But the value of $\mathrm{Y}^{*}$ increases as well, which might become a cause for concern in certain cases. Now keeping $\gamma$ fixed, the value of $\theta$ is varied (Fig. 3 and Fig. 4). Increase of $\theta$ means use of more potent pesticides. This allows $\mathrm{X}^{*}$ to go on increasing with $\theta$ till the equilibrium point attained by the system changes from $E^{*}$ to $E_{A}$. Corresponding to this, the value of $Y$ attained by the system changes from $Y^{*}$ to 0 . If the extreme cases that lead to extinction can be avoided, then use of chemicals can definitely produce good results.

Interestingly, the graphs of both $X(t)$ and $Y(t)$ vs.t display convergent oscillations that stabilizes over time to either $E_{A}$ or $E^{*}$. This shows that immediately after application of chemicals, the production of crops fluctuate slightly and then peaks to a high value. This is followed by several points of high production, which are separated by points of low productivity. The model shows that production keeps fluctuating after application of chemicals. But, if suitable values of $\gamma$ and $\theta$ can be maintained (as mentioned in Section 8 ), then the system reaches a stable state that is more productive and sustainable. 
It has been seen that whenever $E^{*}$ exists, $E_{A}$ also exists. Moreover, existence of $E^{*}$ ensures local asymptotic stability of the system around $E^{*}$. It has also been established that existence of $E^{*}$ guarantees global asymptotic stability of the system and the system's trajectories in the phase plane converge towards $E^{*}$ (Fig. 5). Moreover, existence of $E^{*}$ ensures permanence of the system. Thus, the two conditions,

$2 a /(a+2)>\theta$ and $1-h / r(1+\gamma)>d_{1} /[K \beta\{a(1-\theta / 2)-\theta\}]>0$, are of extreme importance.

Physically, it means moderate value of $\gamma$ and comparatively lower values of $\theta$ is ideal for an agricultural system. Along with these, if the conditions stated in Section 8 are satisfied, we obtain a sustainable and productive agricultural system under the action of chemical agents. The net crop output in this system will be higher than what is possible in the natural system, that is, the system without any chemicals. Also the abundance of pests will be lesser than what is witnessed in the natural system. Thus grazing pressure of the predators on the pests is successfully reduced without making the predators extinct. The model and its analysis establish the effectiveness of chemical intensive farming in areas where pests have advantage of climate, habitat, etc. over the crop plants. The parameter bounds derived in the paper guarantee conservation of the ecological balance. Thus judicious use of chemicals having appropriate potency values can help us expand the land under cultivation - chemical agents can turn areas currently unfit for cultivation due to pest hazards into arable land within a short time frame.

The same model and its associated analysis can be extended beyond agricultural systems. Fisheries provide an ideal situation with the fish being the prey and the larger fish being the predators. Another area of application is the traditional shrimp farming carried out in coastal areas. It is extremely sensitive to pest infestation. Use of suitable chemicals that adversely affect the predators and enhance the growth of target species' can lead to sustainable, productive and profitable systems.

\section{REFERENCES}

[1] Anderson RM, May R, The invasion, persistence and spread of infectious diseases within animal and plant communities, Philos. trans.R. Soc. Lond. B314 (1986), 533-570

[2] Bhattacharya S, Bhattacharya DK, Pest control through viral disease :Mathematical modelling and analysis, J Theor Biol 238 (2006) 177-197

[3] Birkho_ G, Rota GC, Ordinary Differential Equations (Ginn, Boston,1982)

[4] Carson, R., Silent Spring(Houghton Mi_in, Boston, 1962)

[5] Chattopadhyay J, Arino O , A predator-prey model with disease in prey, Nonlinear Analysis, 36 (1999) 747-766

[6] Costello W, Taylor H, Mathematical models of the sterile male technique of insect control, Lecture Notes in Bio-mathematics-5,Springer, New York, (1975), pp. 318-359

[7] Ferrara M, A note on the Solow economic growth mode with Richards population growth law, Applied Sciences, Vol 13 (2011), pp. 36-39 
[8] Greenpeace International, Website :

http://www.greenpeace.org/international/, (Link : seafood/what-is-a-sustainable-_shery)

[9] Hsieh YH, Predator-prey model with disease infection in both populations, Math. Med. Biol., 25 247-266

[10] Krasnov ML, Kiselev AI, Makarenko GI, Functions of a Complex Variable, Operational Calculus, and Stability Theory (Mir Publishers, Moscow, 1984)

[11] Kot M, Elements of Mathematical Ecology(Cambridge University Press, (2001)

[12] Pal AK, Samamnta GP, Stability analysis of an eco-epidemiological model incorporating a prey refuge, Nonlinear Analysis:Modeling and Control. 2010, Vol. 15, No.4, (2010) 473491

[13] Pathak S, Maiti A, Microbial Pest Control : A mathematical model, J of Bio Syst, Vol 18, No. 2 (2010) 455-478

[14] Prout $\mathrm{T}$, The joint effect of release of sterile males and immigration of fertilized females on a density regulated population, J Popul Biol 13(1978) 40-71

[15] Plant ER, Magel M, Modeling and simulation in agricultural pest management, SIAM rev 29: (1987) 235-262

[16] Strogatz SH, Nonlinear Dynamics and Chaos with applications to Physics, Biology, Chemistry and Engineering (Addison-Wesley Publishing Company, 1994)

[17] Tang SY, Chen LS , Modeling and analysis of integrated pest management strategy, Discr Cont Dynam Syst B 4 (2004): 759-768

[18] Zhang H, Chen LS , Pest management through continuous and impulsive control strategies, BioSystems 90 (2007) 350-361 